\title{
THEORETICAL SOLUTION OF PILING COMPACTION AND THE INFLUENCE OF PILE-SOIL-BOUNDARY CURVE HYPOTHESIS
}

\author{
Zi-kun Gao \\ University of Lishui, Faculty of Civil Engineering Department, Li-shui Zhe-jiang \\ 323000,China; gaozikun205@126.com
}

\begin{abstract}
Research is ongoing to find theoretical solution to three-dimensional piling compaction. Considering the spacial-axis-symmetric characteristics, the boundary surface of pile-soil interaction is expressed by polynomials of different orders. First, the curve family parameter is introduced to construct the displacement and integral function. Then, the solution of pile-soil interaction is derived by combining the constitutive relation model of Duncan-Chang and the variational theory. Results of engineering computing show that the theoretical solution converges to the classical CEM and the limit equilibrium theory well at the corresponding computing area. Moreover, the effects of polynomial of different orders on the calculation results are not obvious. The conclusion in this paper can be used for reference in the derivation and application for other interaction of structure and soil problems.
\end{abstract}

\section{KEYWORDS}

Theoretical solution, Piling compaction, Boundary curve hypothesis, Variational method, Integral function

\section{INTRODUCTION}

For cavity expansion, a source-sink [1,2] method is proposed to solve the displacement field caused by pile-soil interaction. Displacement method [3,4]is also applied to solve the problem of cavity expansion in semi-infinite space. With the truth that the ground must be free or zero stress surface for semi-infinite space, the stress superposition [5] is adopted to correct the source-sink method. The above studies should have the following limitations:

(1) Assumption of linear elasticity constitutive model of nonlinear material.

(2) The superposition principle is used to solve the nonlinear displacement field.

\section{METHODS}

In this paper, three-dimensional piling compaction in half-space is researched to solve finite length pile-jacked problem. Firstly, quadratic and quartic polynomial functions are used to simulate the boundary curve respectively. Secondly, combining Duncan-Chang constitutive relation and variational theory, solution is derived for pile-soil interaction in semi-infinite space. Finally, the theoretical result is verified by analyzing the influence of initial boundary curve setting.

The computing workloads of functional integration under two kinds of boundary conditions are compared, and the influence of order of boundary curve on the calculation accuracy and convergence rate is also analyzed. 


\section{MATHEMATICAL MODELS}

Pile-soil interaction is characterized by three-dimensional and strong nonlinearity. Based on the variational theory, the integral functional $[6,7]$ is given as Equation (1).

When the displacement function satisfies all displacement constraints and Equation (1) takes the extreme value, $u_{i}$ will be the theoretical displacement solutions.

$$
\Pi=\iiint_{V}\left[A\left(\varepsilon_{i j}\right)-F_{i} u_{i}\right] \mathrm{d} V-\iint_{S_{1}} \bar{P}_{i} u_{i} \mathrm{~d} S
$$

In the above equation, $F_{i}$ is the volume force, $S_{1}$ is the boundary of the known force, and $\bar{P}_{i}$ is the known force on the boundary; $A\left(\varepsilon_{i j}\right)$ is the potential energy density.

The density of the pile-soil interaction can be given as Equation (2)

$$
A\left(\varepsilon_{i j}\right)=\int_{0}^{\varepsilon_{r}} \sigma_{r}\left(\varepsilon_{i j}\right) \mathrm{d} \varepsilon_{r}+\int_{0}^{\varepsilon_{\theta}} \sigma_{\theta}\left(\varepsilon_{i j}\right) \mathrm{d} \varepsilon_{\theta}+\int_{0}^{\varepsilon_{z}} \sigma_{z}\left(\varepsilon_{i j}\right) \mathrm{d} \varepsilon_{z}+\int_{0}^{\gamma_{z r}} \tau_{z r}\left(\varepsilon_{i j}\right) \mathrm{d} \gamma_{z r}
$$

where $\varepsilon_{i j}$ refers to four strain components $\varepsilon_{r}, \varepsilon_{\theta}, \varepsilon_{z}, \gamma_{z r}$.

Displacement boundary condition is given as $\left.u\right|_{g(z, r)=0}=\bar{u}(r, z)$.

where $\bar{u}(r, z)$ is the displacement value on the known displacement boundary, and $g(z, r)=0$ is the boundary curve equation of the known displacement expressed as Equation (3).

\section{SETTING DISPLACEMENT FUNCTION}

The interaction between pile and the soil around it in semi-infinite space can be regarded as a spatial axisymmetric problem. The mechanical components are independent of the coordinates $\theta$ and functions of the coordinates $(r, z)$. The equation of boundary curve is given as Equation (3) and depicted in Figures 1-2.

Boundary curve of Equation (3) should satisfy, if $z=0, r=r_{0}$ and if $z=H, r=0$, where $H$ is the pile length and $r_{0}$ is the radius of the pile hole at the ground surface.

$$
g(z, r)=f(z, r, H)=0
$$

In order to set the displacement function $u_{r}, w$ to satisfy the known displacement boundary condition, the curve family parameter $z_{0}$ is introduced, as shown in Figures 1-2.

The equation of the curves family with $z_{0}$ is established as shown in Equation (4). The soil around the pile is covered by a series of curves with different values of $z_{0}$. When $z_{0} \geq H$, Eq. (4) can cover any spatial point in the calculation range.

$$
f\left(z, r, z_{0}\right)=0
$$

Equation (4) satisfies $\left.f\left(z, r, z_{0}\right)\right|_{z_{0}=H}=g(z, r)=0 . \partial f /\left.\partial r\right|_{r=0, z \geq H}=0$ 


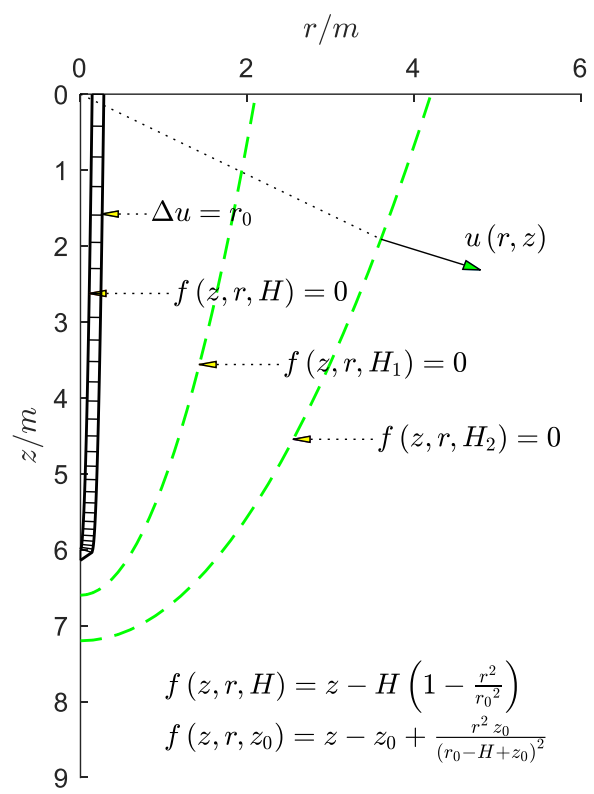

Fig. 1 - Quadratic polynomial piling compaction

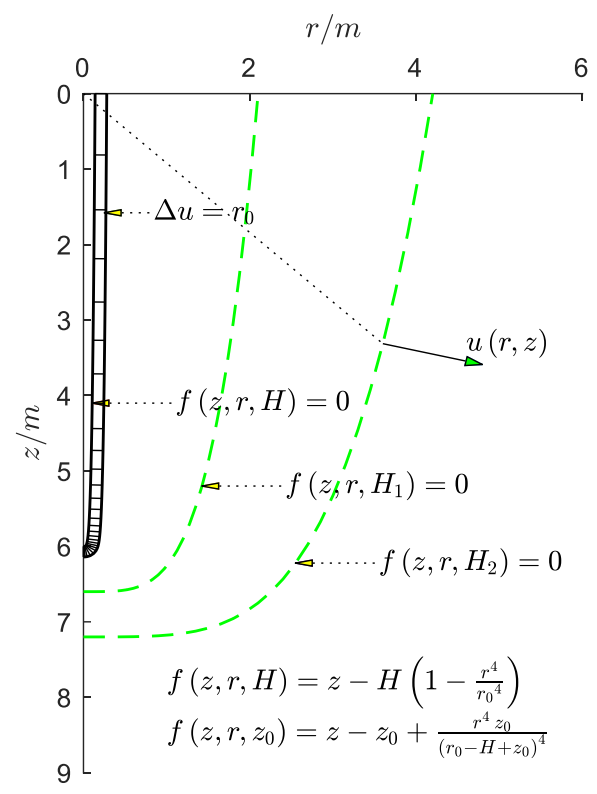

Fig. 2 - Quartic polynomial piling compaction

According to the above analysis, the displacement function is set as Equation (5)

$$
u_{r}=u_{r 0}+\sum_{m} A_{m} u_{r m}, w=w_{0}+\sum_{m} B_{m} w_{m}
$$

where $u_{r 0}=u_{0} M_{0} \sin \alpha, w_{0}=u_{0} M_{0} \cos \alpha, M_{0}=r_{0} /\left(z_{0}-H+r_{0}\right), u_{r m}=u_{0}\left(M_{0} \sin \alpha\right)\left(1-H / z_{0}\right)^{m}$, $w_{m}=u_{0}\left(M_{0} \cos \alpha\right)\left(1-H / z_{0}\right)^{m}, \quad m=1,2,3, \mathrm{~L}$.

$\alpha$ is the angle between outward normal and coordinate line $z$ for the points on the curve $z_{0}$. $\cos \alpha, \sin \alpha$ can be expressed as Equation (6)

$$
\cos \alpha=\frac{f_{z}}{\sqrt{f_{z}^{2}+f_{r}^{2}}}, \sin \alpha=\frac{f_{r}}{\sqrt{f_{z}^{2}+f_{r}^{2}}}
$$

where $f_{z}=\frac{\partial f\left(z, r, z_{0}\right)}{\partial z}, f_{r}=\frac{\partial f\left(z, r, z_{0}\right)}{\partial r} . A_{m}, B_{m}$ are $2 m$ undetermined coefficients.

$z_{0}=H$ represents the boundary curve of the hole wall. When $z_{0}=H, 1-H / z_{0}=0$, the displacement function satisfies the boundary condition of the hole wall. 


\section{GEOMETRIC AND CONSTITUTIVE EQUATIONS}

Since the parameters $z_{0}$ are introduced into the displacement function, the Straindisplacement relationship are given as Equation (7)

$$
\left\{\begin{array}{l}
\varepsilon_{r}=-\left(\frac{\partial u_{r}}{\partial r}+\frac{\partial u_{r}}{\partial z_{0}} \frac{\partial z_{0}}{\partial r}\right), \varepsilon_{z}=-\left(\frac{\partial w}{\partial z}+\frac{\partial w}{\partial z_{0}} \frac{\partial z_{0}}{\partial z}\right) \\
\gamma_{z r}=\frac{\partial u_{r}}{\partial z}+\frac{\partial u_{r}}{\partial z_{0}} \frac{\partial z_{0}}{\partial z}+\frac{\partial w}{\partial r}+\frac{\partial w}{\partial z_{0}} \frac{\partial z_{0}}{\partial r}, \varepsilon_{\theta}=-\frac{u_{r}}{r}
\end{array}\right.
$$

where $\frac{\partial z_{0}}{\partial r}=-\frac{f_{r}\left(z, r, z_{0}\right)}{f_{z_{0}}\left(z, r, z_{0}\right)}, \frac{\partial z_{0}}{\partial z}=-\frac{f_{z}\left(z, r, z_{0}\right)}{f_{z_{0}}\left(z, r, z_{0}\right)}, f_{z 0}=\frac{\partial f\left(z, r, z_{0}\right)}{\partial z_{0}}$.

Duncan-Chang model is adopted [8,9], as shown in Equation (8) and (9). The relationship curve between stress and primary strain of the constitutive model is shown in Figure 3.

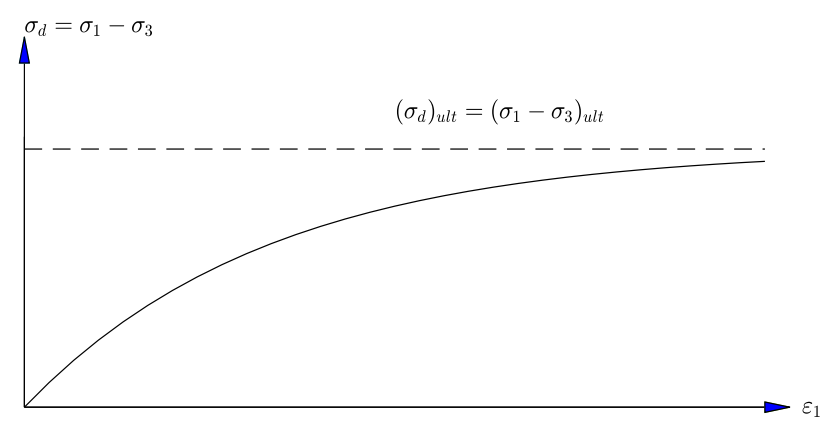

Fig. 3 - Duncan - Chang curve $\sigma_{d} \sim \varepsilon_{1}$

$$
\sigma_{d}=\varepsilon_{1} /\left[\frac{1}{E_{i}}+\frac{\varepsilon_{1}}{\left(\sigma_{d}\right)_{f}} R_{f}\right]
$$

Modulus of elasticity is given as

$$
E_{i}=K p_{a}\left(\sigma_{3} / p_{a}\right)^{n}
$$

where, $\quad\left(\sigma_{d}\right)_{f}=\left(2 c \cos \varphi+2 \sigma_{3} \sin \varphi\right) /(1-\sin \varphi) \quad R_{f}=\left(\sigma_{d}\right)_{f} /\left(\sigma_{d}\right)_{u l t}$ is failure ratio. $c, \varphi$ is cohesion and internal friction angle of the soil, $p_{a}$ is the normal atmospheric pressure, $\sigma_{3}$ is preconsolidation pressure, and $K, n$ are the experimental constant.

Equation (8) can be used to derive nonlinear secant modulus by Equation (10) 


$$
E_{\mathrm{sec}}=\frac{\sigma_{d}}{\varepsilon_{1}}=\frac{1}{\frac{1}{E_{i}}+\frac{\varepsilon_{1}}{\left(\sigma_{d}\right)_{f}} R_{f}}
$$

where $\varepsilon_{1}$ is the first main strain [10], determined by Equation (11).

$$
\varepsilon_{1}=\frac{\varepsilon_{r}+\varepsilon_{z}}{2} \pm \sqrt{\frac{\left(\varepsilon_{r}-\varepsilon_{z}\right)^{2}}{4}+\frac{\gamma_{z r}^{2}}{4}}
$$

The volume deformation modulus of Duncan-Chang model is expressed as Equation (12)

$$
K_{t}=K_{b} p_{a}\left(\sigma_{3} / p_{a}\right)^{m_{0}}
$$

where, $K_{b}, m_{0}$ is the experimental constant.

\section{POTENTIAL ENERGY DENSITY}

The increment of stress components [11] can be expressed as Equation (13)

$$
\left\{\begin{array}{l}
\Delta \sigma_{r}=T_{1}\left[T_{2}\left(\varepsilon_{r}+\varepsilon_{\theta}+\varepsilon_{z}\right)+\varepsilon_{r}\right], \Delta \sigma_{\theta}=T_{1}\left[T_{2}\left(\varepsilon_{r}+\varepsilon_{\theta}+\varepsilon_{z}\right)+\varepsilon_{\theta}\right] \\
\Delta \sigma_{z}=T_{1}\left[T_{2}\left(\varepsilon_{r}+\varepsilon_{\theta}+\varepsilon_{z}\right)+\varepsilon_{z}\right], \Delta \tau_{z r}=T_{1} \gamma_{z r} / 2
\end{array}\right.
$$

where $T_{1}=E_{\mathrm{sec}} /\left(1+v_{0}\right), T_{2}=v_{0} /\left(1-2 v_{0}\right)$,

$\varepsilon_{r}, \varepsilon_{\theta}, \varepsilon_{z}, \tau_{r z}$ is determined by Equation (7), $v_{0}$ is Poisson ratio. According to Equation (13), the total stress can be expressed as

$$
\sigma_{r}=\Delta \sigma_{r}+\frac{v_{0}}{1-v_{0}} \gamma z, \sigma_{\theta}=\Delta \sigma_{\theta}+\frac{v_{0}}{1-v_{0}} \gamma z, \sigma_{z}=\Delta \sigma_{z}+\gamma z, \tau_{z r}=\Delta \tau_{z r}
$$

where $\gamma$ is the buoyant unit weight, $\gamma z$ is the consolidation stress. Therefore, the potential energy density can be expressed as:

$$
A\left(\varepsilon_{i j}\right)=\int_{0}^{\Delta u}\left[\sigma_{r} \frac{\partial \varepsilon_{r}}{\partial u_{0}}+\sigma_{\theta} \frac{\partial \varepsilon_{\theta}}{\partial u_{0}}+\sigma_{z} \frac{\partial \varepsilon_{z}}{\partial u_{0}}+\tau_{z r} \frac{\partial \gamma_{z r}}{\partial u_{0}}\right] \mathrm{d} u_{0}
$$

Since the curve parameter $z_{0}$ is introduced, the potential energy density can be written as Equation (14), which is a function of $z, r, z_{0}$ as

$$
A\left(\varepsilon_{i j}\right)=\int_{0}^{\Delta u} D\left(z, r, z_{0}, u_{0}, \bar{A}, \bar{B}\right) \mathrm{d} u_{0}
$$

where $z, r, z_{0}$ meet the constraint conditions of $f\left(z, r, z_{0}\right)=0, \bar{A}=\left\{A_{1}, A_{2}, \ldots, A_{m}\right\}$, $\bar{B}=\left\{B_{1}, B_{2}, \ldots, B_{m}\right\}$, and 


$$
D\left(z, r, z_{0}, u_{0}, \bar{A}, \bar{B}\right)=\sigma_{r}\left(\varepsilon_{i j}\right) \frac{\partial \varepsilon_{r}}{\partial u_{0}}+\sigma_{\theta}\left(\varepsilon_{i j}\right) \frac{\partial \varepsilon_{\theta}}{\partial u_{0}}+\sigma_{z}\left(\varepsilon_{i j}\right) \frac{\partial \varepsilon_{z}}{\partial u_{0}}+\tau_{z r}\left(\varepsilon_{i j}\right) \frac{\partial \gamma_{z r}}{\partial u_{0}}
$$

\section{FUNCTIONAL CONSTRUCTION AND COEFFICIENTS DETERMINATION}

According to energy density of Equation (14), Equation (1) can be written as Equation (15)

$$
\Pi=\iiint_{V} A\left(\varepsilon_{i j}\right) \mathrm{d} V=2 \pi \iint_{S} \int_{0}^{\Delta u} D\left(z, r, z_{0}, u_{0}, \bar{A}, \bar{B}\right) r \mathrm{~d} u_{0} \mathrm{~d} S
$$

where: $A\left(\varepsilon_{i j}\right)$ is represented by Equation (14).

The coefficient $\bar{A}, \bar{B}$ can be solved by the extreme calculation of Equation (16), and the theoretical solution of displacement and stress field can be obtained then.

$$
\frac{\partial \Pi}{\partial A_{i}}=0, \quad \frac{\partial \Pi}{\partial B_{i}}=0
$$

\section{PROJECT CASES}

Equation (3) is set as the polynomial function as shown in Equation (17) - (18)

$$
\begin{aligned}
& z=H\left(1-r^{2} / r_{0}{ }^{2}\right) \\
& z=H\left(1-r^{4} / r_{0}{ }^{4}\right)
\end{aligned}
$$

where $H$ is the pile length, $r_{0}$ is the equivalent radius $[12,13]$ of the pile hole on the ground surface. The equivalent condition of pile hole expansion is established as follows

$$
\pi\left[\left(2 r_{0}\right)^{2}-r_{0}^{2}\right]=\pi R_{0}^{2} \Rightarrow r_{0}=R_{0} / \sqrt{3}=d /(2 \sqrt{3})
$$

with regard to the pile of $H=6 \mathrm{~m}, d=2 R_{0}=0.5 \mathrm{~m}, r_{0}=d /(2 \sqrt{3})=0.14 \mathrm{~m}$.

Soil parameters are shown in Table 1. Considering the consolidation stress of shallow soil is small and it is relatively loose and has less influence on the calculation result, mean values of muddy clay and clay are calculated and we get $c=14 \mathrm{kPa}, \varphi=13.25^{\circ}$, where $c, \varphi$ are consolidated undrained (CU) parameters.

The constitutive parameters of clay for example 4.1 and 4.2 are [9] $R_{f}=0.8, K=200$, $n=0.5, K_{b}=50, m_{0}=0.5$. 
Tab. 1 - Soil parameters

\begin{tabular}{lllllll}
\hline Soil & Depth & $E_{s}$ & & $\gamma$ & $c$ & \\
& $\mathrm{~m}$ & $\mathrm{MPa}$ & $v_{0}$ & $\mathrm{KN} / \mathrm{m}^{3}$ & $\mathrm{kP}$ & $\varphi$ \\
\hline Silty clay & $0 \sim 2$ & 34 & 0.29 & 18.5 & 12 & $18.0^{\circ}$ \\
Muddy clay & $2 \sim 4$ & 29 & 0.46 & 17.0 & 13 & $12.0^{\circ}$ \\
clay & $4 \sim$ & 32 & 0.42 & 17.5 & 15 & $14.5^{\circ}$ \\
\hline
\end{tabular}

\section{Example 1}

Family curves can be written as Equation (19), as shown in Figure 1,

$$
f\left(z, r, z_{0}\right)=z-z_{0}+\frac{z_{0} r^{2}}{\left(z_{0}-H+r_{0}\right)^{2}}=0
$$

when $z_{0}=H, f\left(z, r, z_{0}\right)=z-H\left(1-r^{2} / r_{0}^{2}\right)$

Equation (20) are given for calculating the stress in the plastic and elastic zone around the pile by the cavity expansion method (CEM) [14,15].

Figure 4 shows a comparative analysis of the calculated results of Equation (20), numerical simulation results [16] and the theoretical solution derived in this paper based on Equation (19).

$$
\begin{gathered}
\Delta \sigma_{r}=2 c_{u} \ln \frac{R_{p}}{r}+c_{u}, \Delta \sigma_{z}=2 c_{u}+\ln \frac{R_{p}}{r} \\
\Delta \sigma_{r}=c_{u}\left(\frac{R_{p}}{r}\right)^{2}, \Delta \sigma_{z}=0
\end{gathered}
$$

In the above equation, $R_{p}=R_{0} \sqrt{E /\left[2(1+\mu) c_{u}\right]}$ is the radius of the plastic zone, $c_{u}\left(c_{u} \geq c\right)$ is the index of unconsolidated undrained (UU), and this article takes $c_{u}=c . R_{0}$ is the radius of pile hole.

Equation (21) is given for calculating the stress in the $r$ direction based on the limit equilibrium theory [17]. Figure 5 shows a comparative analysis of the lateral pressure results based on the limit equilibrium theory and the theoretical solution derived in this paper based on Equation (19).

$$
\sigma_{r}=\gamma z\left[\tan ^{2}\left(\frac{\pi}{4}+\frac{\varphi}{2}\right)\right]+2 c \tan \left(\frac{\pi}{4}+\frac{\varphi}{2}\right)
$$

Figure 4 shows that calculated results of the theoretical solution are basically matching with those of the cavity expansion method at the zone located in the region of $r \geq 1 d \& z \leq 6 \mathrm{~m}$. Since the cavity expansion method (CEM) assumes that the expansion model satisfies the plane strain 
condition, it is only suitable for solving the plane strain problem. For finite-length pile threedimensional expansion, the region below or around the pile tip is obviously inconsistent with the assumption of the plane strain. Compared with the CEM results, the theoretical solution of this paper is more reasonable with the rapidly decreasing of radial pressure around or below the pile tip.

Figure 5 shows that the results of theoretical solution are basically matching with the results of limit equilibrium theory at plastic line located in the range of $0 \mathrm{~m} \leq z \leq 6 \mathrm{~m} \& \quad r=0.25 \mathrm{~m}$. Compared with the limit equilibrium results, the theoretical solution of this paper is more reasonable, with the rapidly decreasing of radial pressure around or below the pile tip. In addition, because the limit equilibrium results are directly related to the volume-weight of the soil, the curve of passive earth pressure shown in Figure 5 has inflection points at the interface of the soil layer.

Figure 5 also shows that the results of theoretical solution are basically matching with the results of numerical simulation results at $r=0.75 \mathrm{~m}$.

\section{Example 2}

Family curves can be written as Equation (22), as shown in Figure 2

$$
f\left(z, r, z_{0}\right)=z-z_{0}+\frac{z_{0} r^{4}}{\left(z_{0}-H+r_{0}\right)^{4}}=0
$$

when $z_{0}=H, f\left(z, r, z_{0}\right)=z-H\left(1-r^{4} / r_{0}^{4}\right)$

Figures 6-7 can be obtained, in combination with the theoretical solution derived in this paper based on Equation (22), the results of numerical simulation results, CEM and limit equilibrium theory.

The stress regularities of distribution in Figures 6-7 are basically the same as those in Figures 4-5. They explain that the quadratic or quartic polynomial of the boundary curve has little effect on the calculation results, because the soil near the pile-soil interaction boundary has been in the plastic yield state already.

To sum up, with the increase of undetermined coefficients from 0 to 4 , the additional stress generated by pile compaction rapidly converges to the reasonable value and polynomial with lower order can also be used to simulate the initial hole wall boundary to reduce the computational workload. 
CIVIL

ENGINEERING

\section{JOURNAL}

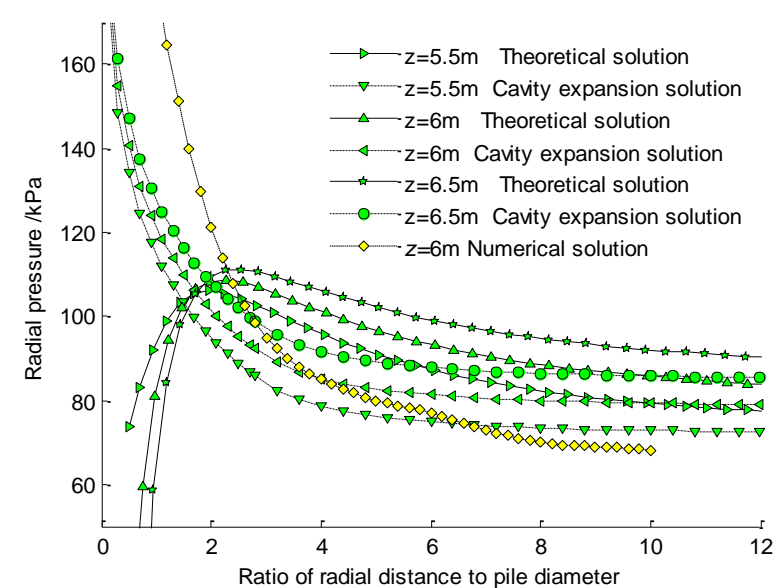

(a) Displacement function with 0 undetermined coefficient

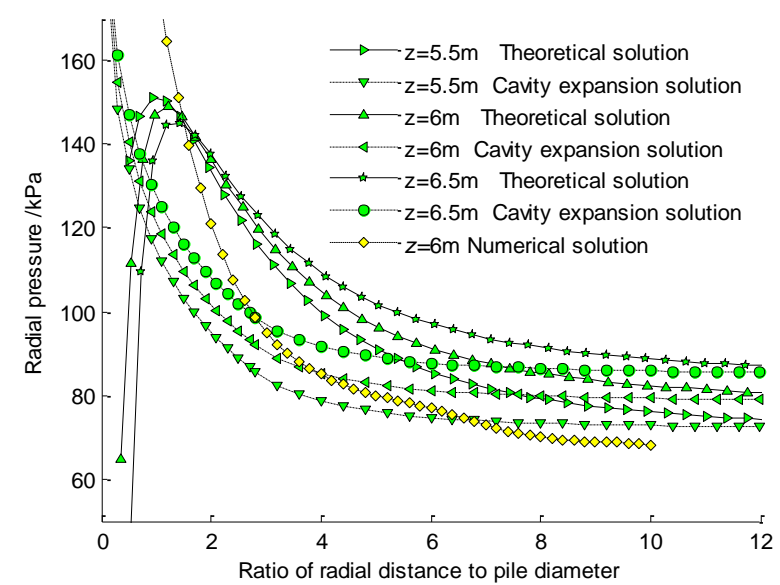

(b) Displacement function with 2 undetermined coefficient

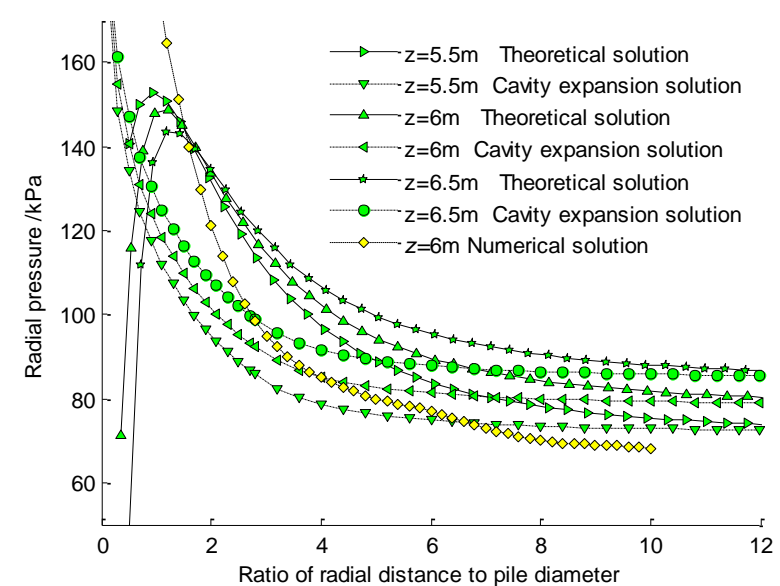

(c) Displacement function with 4 undetermined coefficient

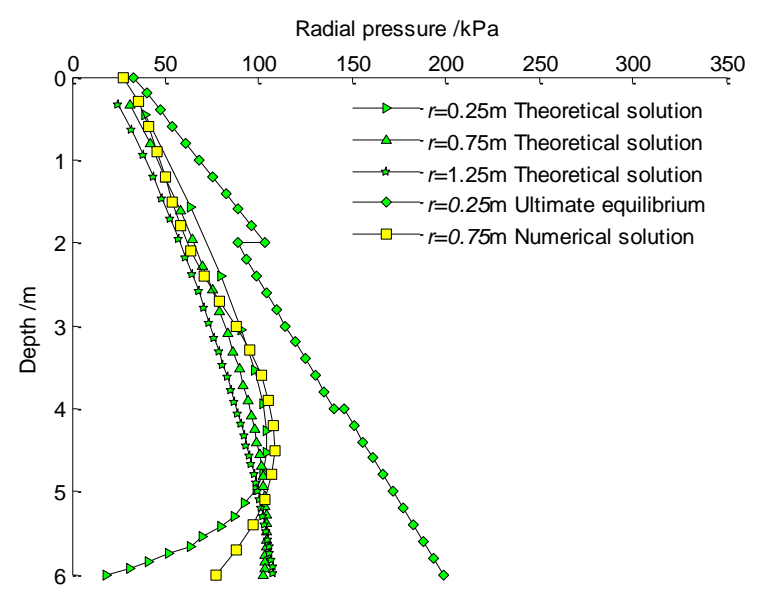

(a) Displacement function with 0 undetermined coefficient

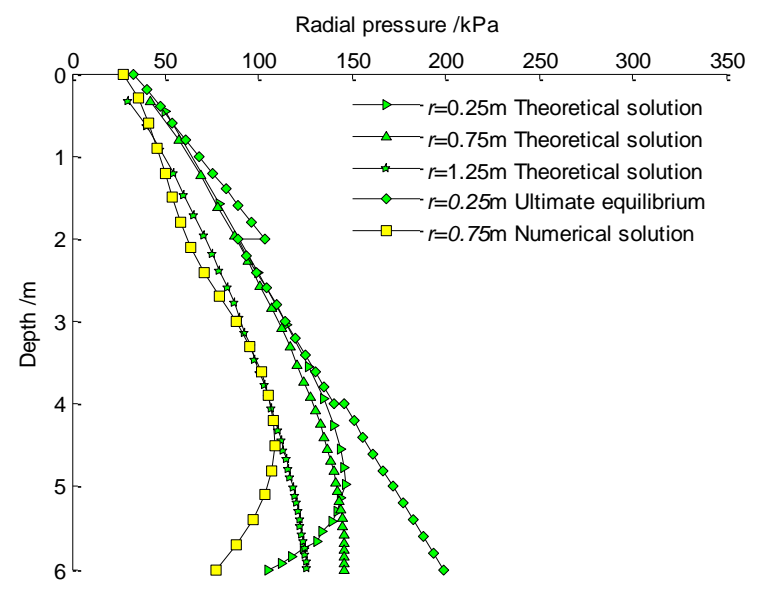

(b) Displacement function with 2 undetermined coefficients

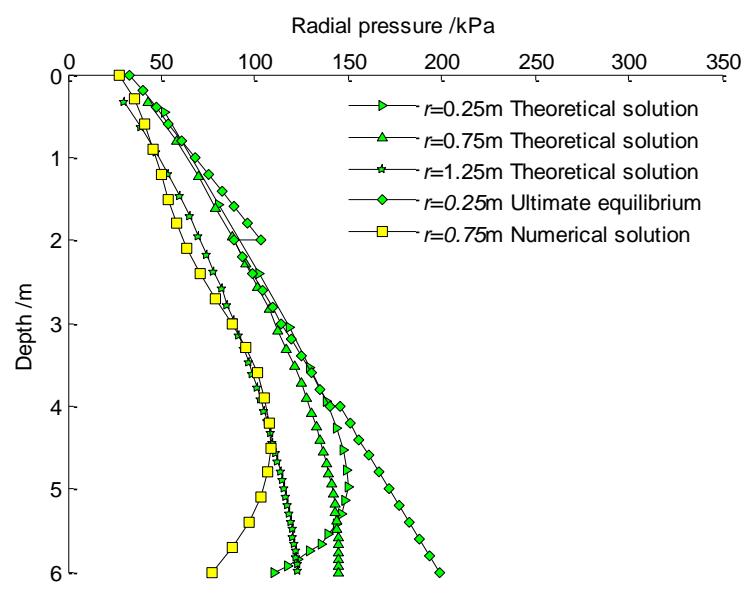

(c) Displacement function with 4 undetermined coefficients

Fig. 5 - The radial pressure along depth

Fig. 4 - The radial pressure along radial direction 
CIVIL

ENGINEERING

THE CIVIL ENGINEERING JOURNAL 1-2021 JOURNAL

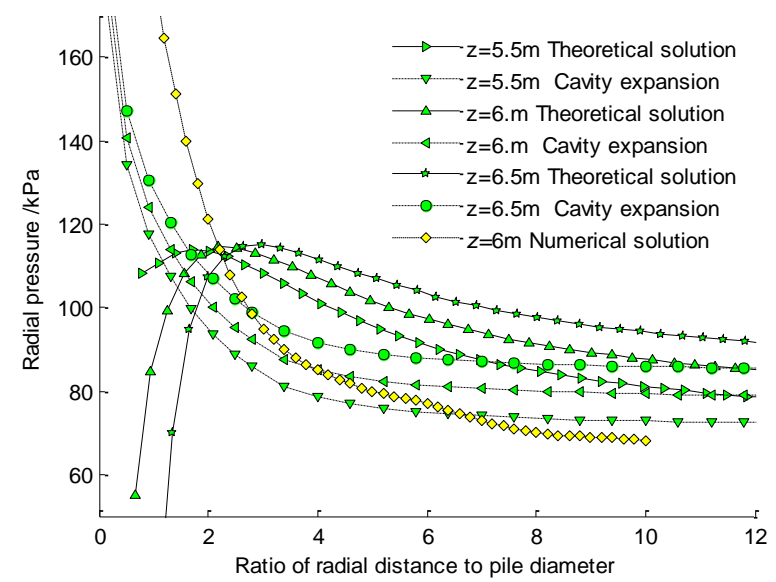

(a) Displacement function with 0 undetermined coefficient

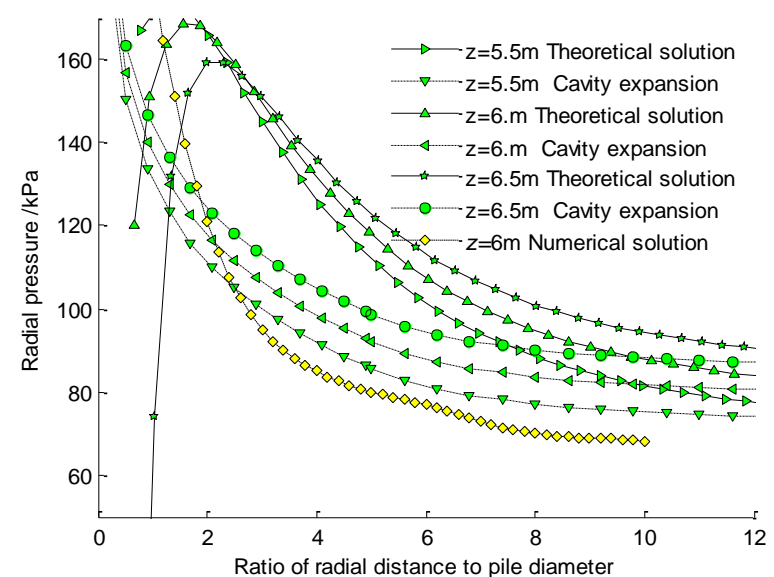

(b) Displacement function with 2 undetermined coefficient

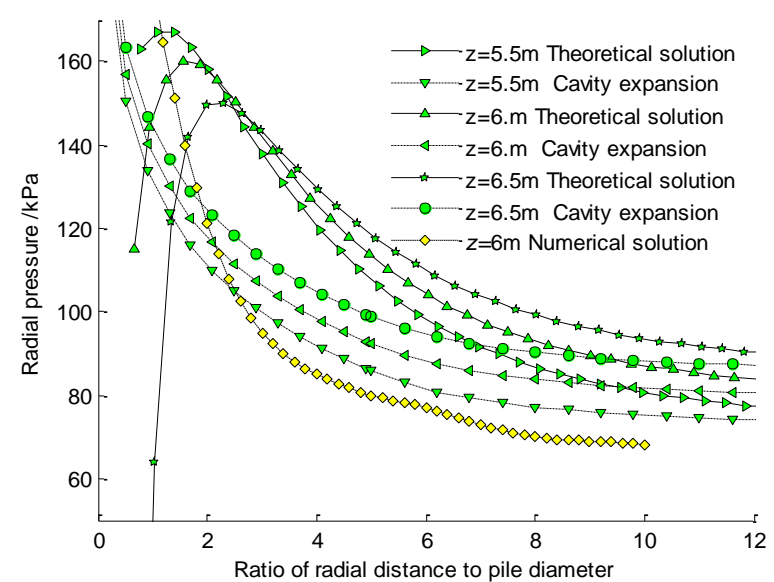

(c) Displacement function with 4 undetermined coefficient

Fig. 6 - The radial pressure along radial direction

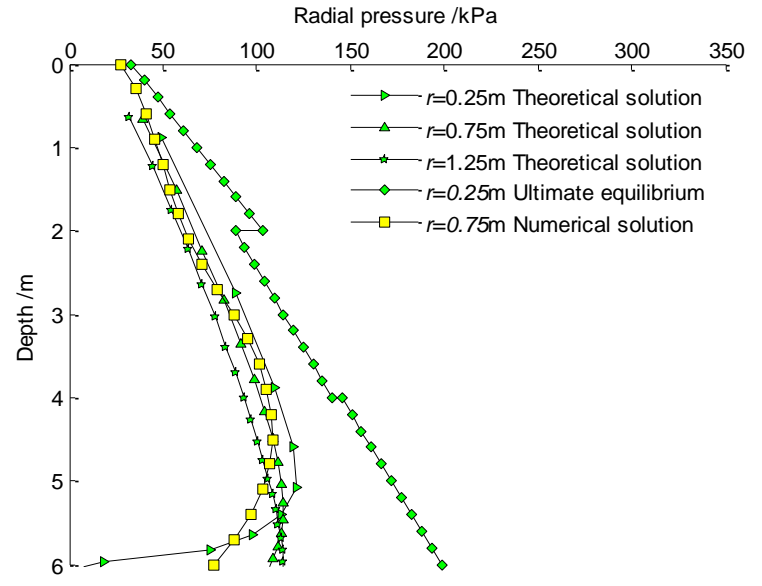

(a) Displacement function with 0 undetermined coefficient

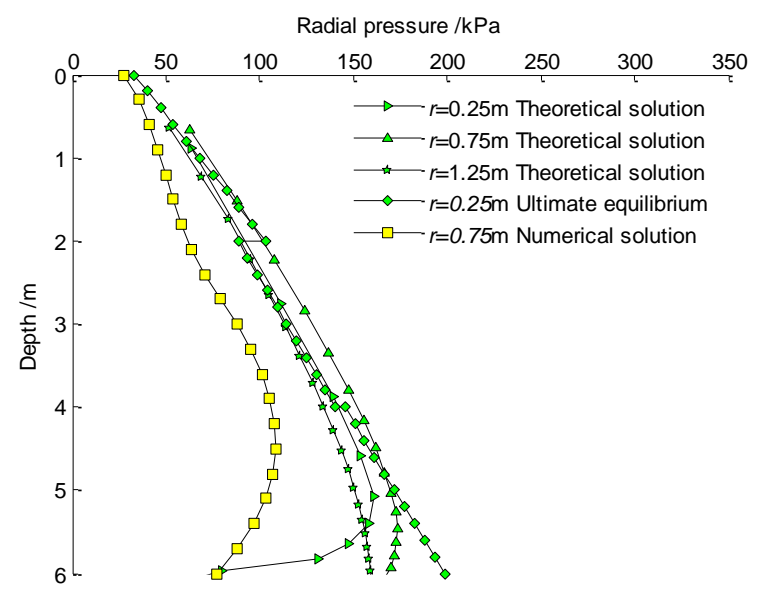

(b) Displacement function with 2 undetermined coefficients

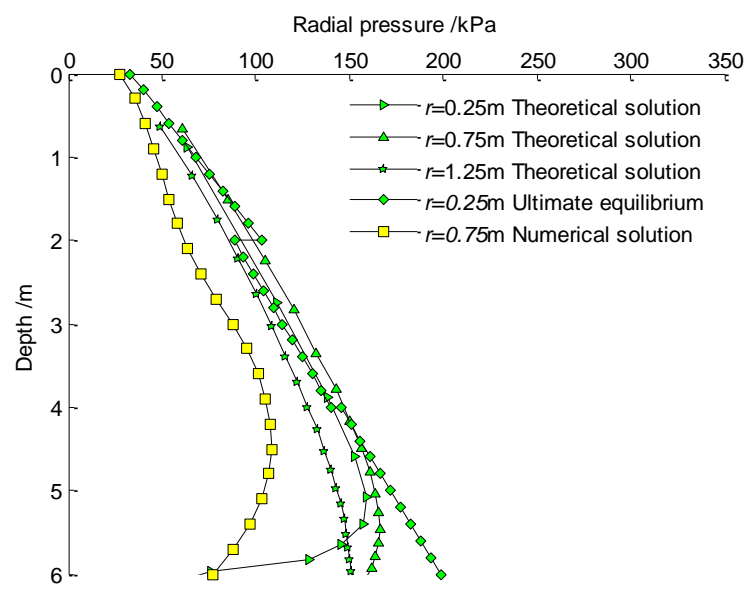

(c) Displacement function with 4 undetermined coefficients

Fig. 7 - The radial pressure along depth 


\section{Example 3}

The test site is located in a section of Taiwan high-speed railway project [18]. The precast concrete pile length is $34 \mathrm{~m}$, and diameter is $80 \mathrm{~cm}$. The distribution of the test pile and measuring pipes are shown in Figure 8. Burial depth of survey tubes are $40 \mathrm{~m}$. The soil layers in the site are inter-bedded sandy soil and cohesive soil, and the critical values of sandy and clay are taken as parameters for calculating. Parameters of soil layer $[9,18]$ are shown in Table 2 . The equivalent pile-hole-expansion radius is $r_{0}=0.4 / \sqrt{3}=0.23 \mathrm{~m}$, and the normal displacement of the hole wall is $\Delta u=r_{0}=0.23 \mathrm{~m}$.

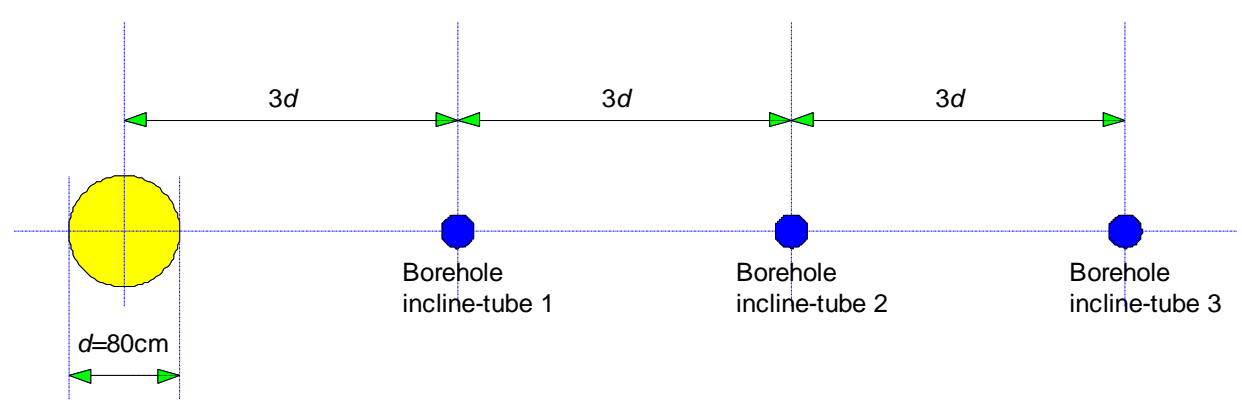

Fig. 8 - Test pile and plane layout of incline-tubes

Tab. 2 - Geotechnical parameters of test site

\begin{tabular}{cccccccc}
\hline Cohesion & Friction Angle & Poisson's ratio & Soil bulk density & Destructive & ratio & Constant & Constant \\
$c / \mathrm{kPa}$ & $\varphi /{ }^{\circ}$ & $v$ & $\gamma / \mathrm{kPa}$ & $R_{f}$ & $K$ & $n$ \\
\hline 3 & 20 & 0.3 & 18 & 0.9 & 300 & 0.5 \\
\hline
\end{tabular}

Displacement Equation (23 26) can be deduced based on Equation (16 17, 19), and Figure 9-10 can be obtained in combination with the theoretical solution derived in this paper and field test data in reference [18]. Figure 9 shows distribution-regularities comparison between theoretical computing radial displacement and the in-situ measured data at different penetration depth. Figure 10 shows comparison between theoretical displacement results and the in-situ measured data at different coordinate $r$. Figure 9 shows that for different penetration depth, the calculated value of displacement is basically agreement with the in-situ measured value, although the theoretical calculation cannot accurately reflect the characteristics due to injection interval. Figure 10 shows that for different radial coordinates, the calculated value is basically agreement with the measured value.

When Penetration depth: $H=9 \mathrm{~m}$, displacement function due to pile compaction can be written as Equation (23)

$$
\left\{\begin{array}{l}
u_{r}=\Delta u\left(\frac{r_{0}}{z_{0}-H+r_{0}} \sin \alpha\right)\left[1-1.0\left(1-\frac{H}{z_{0}}\right)\right] \\
w=\Delta u\left(\frac{r_{0}}{z_{0}-H+r_{0}} \cos \alpha\right)\left[1-5.0\left(1-\frac{H}{z_{0}}\right)\right]
\end{array}\right.
$$


When Penetration depth: $H=17 \mathrm{~m}$, displacement function due to pile compaction can be written as Equation (24)

$$
\left\{\begin{array}{l}
u_{r}=\Delta u\left(\frac{r_{0}}{z_{0}-H+r_{0}} \sin \alpha\right)\left[1-1.0\left(1-\frac{H}{z_{0}}\right)\right] \\
w=\Delta u\left(\frac{r_{0}}{z_{0}-H+r_{0}} \cos \alpha\right)\left[1-1.7\left(1-\frac{H}{z_{0}}\right)\right]
\end{array}\right.
$$

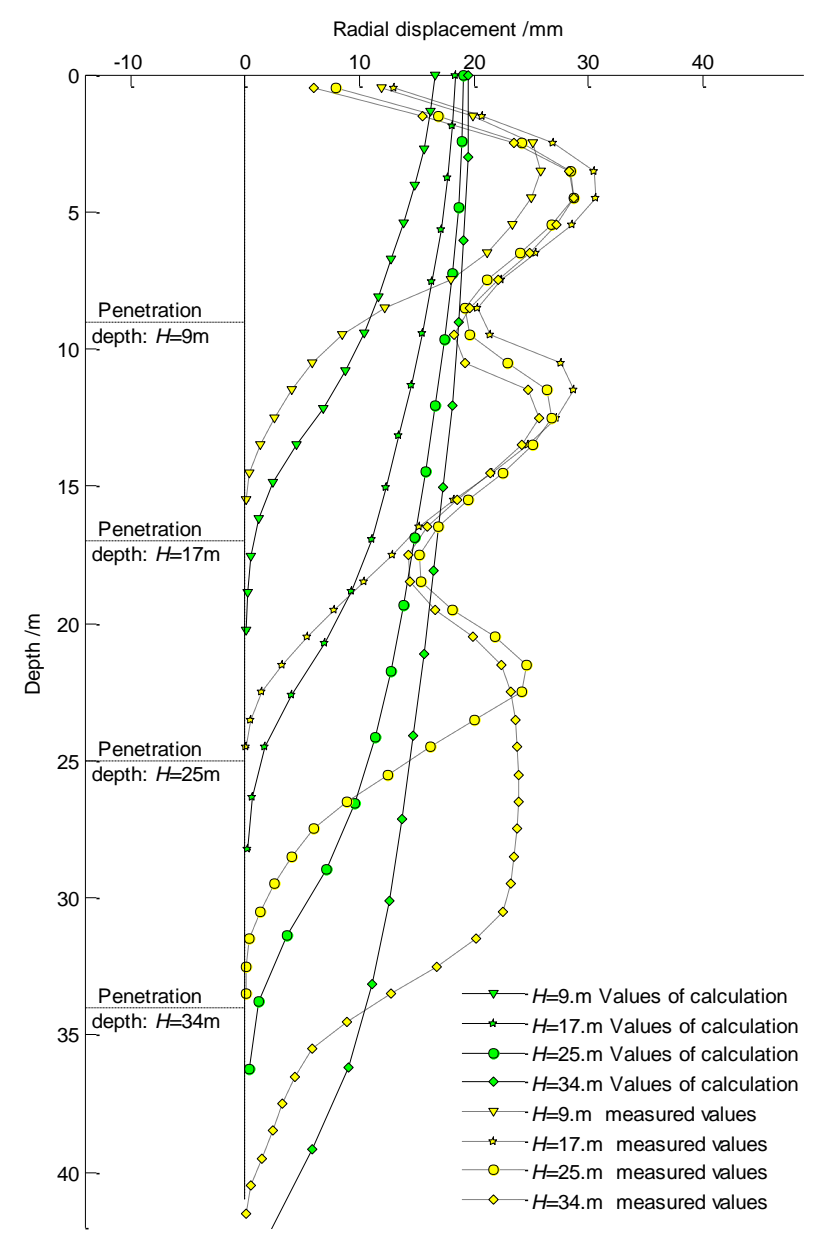

Fig.9 - Displacement at different penetration depth

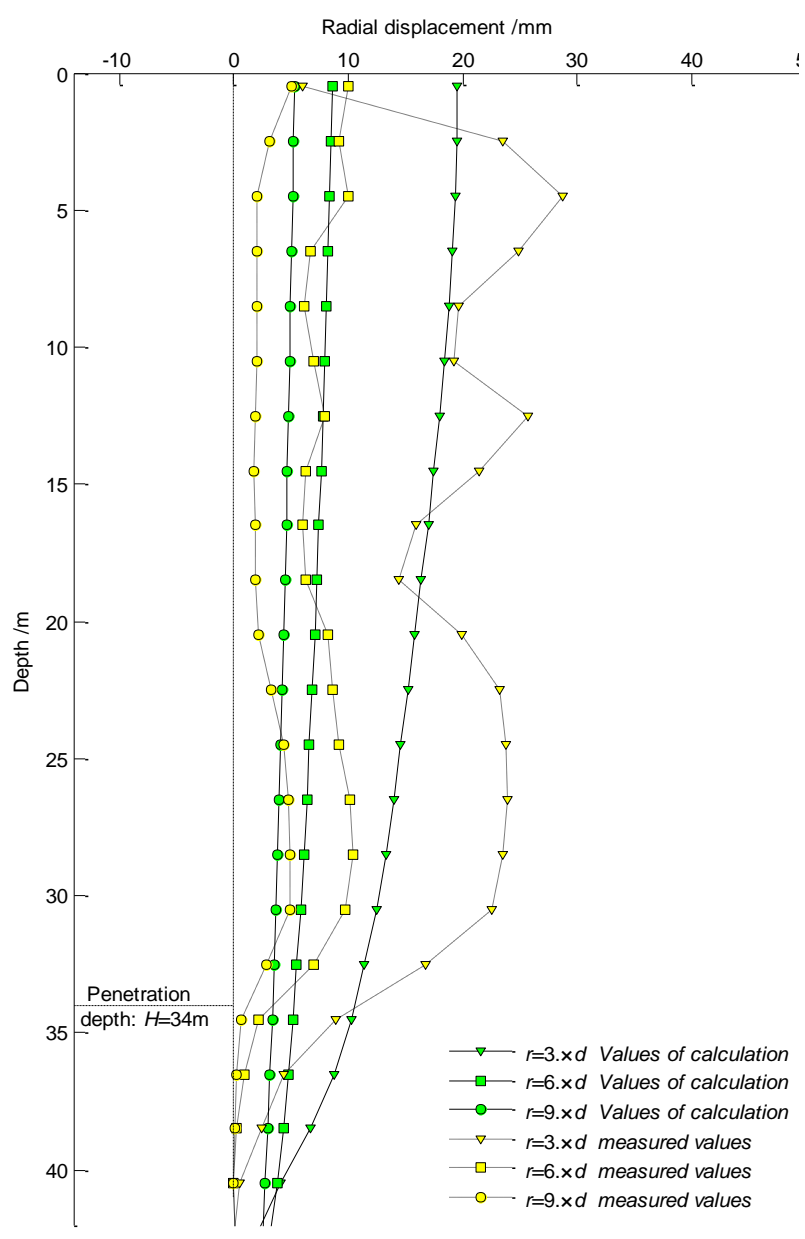

Fig. 10 - Displacement at different $r$

When Penetration depth: $H=25 \mathrm{~m}$, displacement function due to pile compaction can be written as Equation (25) 


$$
\left\{\begin{array}{l}
u_{r}=\Delta u\left(\frac{r_{0}}{z_{0}-H+r_{0}} \sin \alpha\right)\left[1-1.0\left(1-\frac{H}{z_{0}}\right)\right] \\
w=\Delta u\left(\frac{r_{0}}{z_{0}-H+r_{0}} \cos \alpha\right)\left[1-1.1\left(1-\frac{H}{z_{0}}\right)\right]
\end{array}\right.
$$

When Penetration depth: $H=34 \mathrm{~m}$, displacement function due to pile compaction can be written as Equation (26)

$$
\left\{\begin{array}{l}
u_{r}=\Delta u\left(\frac{r_{0}}{z_{0}-H+r_{0}} \sin \alpha\right)\left[1-1.0\left(1-\frac{H}{z_{0}}\right)\right] \\
w=\Delta u\left(\frac{r_{0}}{z_{0}-H+r_{0}} \cos \alpha\right)\left[1-1.1\left(1-\frac{H}{z_{0}}\right)\right]
\end{array}\right.
$$

\section{CONCLUSIONS}

Theoretical solutions of the piling compaction are derived by combining the constitutive relation model of Duncan-Chang and the variational theory. Boundary surface could be expressed by polynomials of different orders for piling compaction, considering the characteristics of pile-soil interaction such as three-dimension and strong nonlinearity.

Computing of the project cases shows that the solutions of variational theory converges to results of cavity expansion and limit equilibrium theory well in the corresponding region. The influences of pile-soil boundary curve on the calculation accuracy and convergence rate are not obvious. Numerical and in-situ test results are also used to verify the reasonable and validity of the deduced theoretical solutions.

Researches in this paper show that the polynomial with lower order can be used to simulate the initial hole wall boundary to reduce the computational workload. 


\section{REFERENCES}

[1] Sagaseta. Analysis of undrained soil deformation due to ground loss[J]. Geotechnique,1987, 37(3): $301-320$

[2] Sagaseta. Prediction of Ground Movements Due to Pile- Driving in Clay [J]. Journal of Geotechnical and Geoenvironmental Engineering. 2001, 127(1): 55-66.

[3] Luo Zhanyou. Study on compacting effects and construction measures of jacked pile [D]. Hangzhou: Zhejiang University, 2004. 19-21 (in Chinese)

[4] Wang Pengcheng. Study on Cavities Expansion in Soils with Softening and Dilation and Analysis of Pile Driving Effects [D]. Hangzhou: Zhejiang Univ. 2005. 175-177 (in Chinese)

[5] Zhu Ning. Theoretical Analysis of Soil Deformation Due to Pile Jacking [D]. Nanjing: Hohai Univ., 2005. 131-132. ( in Chinese)

[6] Niu Yangjun. Modernistic Variation Principle[M]. Beijing: Press of Beijing University of Technology, 1992 (in Chinese)

[7] Wang Xucheng, Shao Min. Numerical method and basic theory of finite element method [M]. Beijing: Tsinghua University Press, 1997.

[8] QIAN J H, YIN Z Z. Principle and calculation of geotechnical engineering[M].2nd ed. Beijing: China Water \& Power Press, 1996(in Chinese).

[9] ZHENG Ying-ren, SHEN Zhu-jiang, GONG Xiao-nan. The principles of geotechnical plastic mechanics[M]. Beijing: China Architecture and Building Press, 2002: 180-183. (in Chinese))

[10] GAO Zi-kun. Theoretical Analysis of Soil Squeezing Effect and Conslidation Characteristic Due to Pile Jacked [D]. Nanjing: Hohai Univ., 2007. 57-66. ( in Chinese)

[11] Xu Zhi-lun. Elastic mechanics[M]. 3rd ed. Beijing: Higher Education Press, 1984: 274-278. ( in Chinese))

[12] Carter, J.P., Randolph, M.F. and Wroth, C.P. (1979). Stress and pore pressure changes in clay during and after the expansion of cylindrical cavity. Int. J. for Num. and Analy. in Geomech. Vol.3, 1979.

[13] RANDOLPH M F, CARTER J P, WROTH C P. Driven piles in clay - the effects of installation and subsequent consolidation[J]. Géotechnique, 1979, 29(4): 361-393.

[14] Visic, A. C., Expansion of Cavity in Infinite Soil Mass, Jour. Soil Mech. Found. Div., A. S. C. E., 1972, 98(3), 265-289.

[15] Atkinson, J. H. and Bransby P. L. The Mechanics of Soils[M].McGraw-Hill, England, 1978.

[16] TANG Shi-dong, LI Yang. Analysis of a driven pile by ANSYS. Rock And Soil Mechanics [J]. Vol. 27 , 2006: 973 976.

[17] Chen Xizhe. Soil mechanics and foundation [M]. Beijing: Tsinghua Press. 2004.

[18] Jin-Hung Hwang, Neng Liang and Cheng-Hsing Chen. Ground Respond During Pile Driving. Journal of Geotechnical and Geoenvironmental Engineering. Nov., 2001: 939 948. 